\title{
A new all-sky catalog of stars with large proper motions
}

\author{
S. Lépine ${ }^{1}$, M. M. Shara ${ }^{1}$, R.-M. Rich ${ }^{2}$, \\ A. Wittenberg ${ }^{1}$, M. Halmo ${ }^{1}$, B. Bongiorno ${ }^{1}$ \\ ${ }^{1}$ Dept. of Astrophysics, American Museum of Natural History, Central Park West at 79th \\ street, New York, NY 10024, USA \\ email: lepine@amnh.org, mshara@amnh.org \\ ${ }^{2}$ Dept. of Astrophysics, University of California at Los Angeles rmr@astro.ucla.edu
}

\begin{abstract}
A new all-sky catalog of stars with proper motions $\mu>0.15^{\prime \prime} \mathrm{yr}^{-1}$ is presented. The catalog is largely a product of the SUPERBLINK survey, a data-mining initiative in which the entire Digitized Sky Surveys are searched for moving stellar sources. Findings from earlier proper motions surveys are also incorporated. The new all-sky catalog supersedes the great historic proper motion catalogs assembled by W. J. Luyten (LHS, NLTT), and provides a virtually complete $>98 \%$ census of high proper motion stars down to magnitude $R=19$.
\end{abstract}

Keywords. catalogs, solar neighborhood, stars: kinematics, stars: low-mass, brown dwarfs, stars: subdwarfs, stars: white dwarfs, Galaxy: stellar content

\section{The SUPERBLINK survey}

We have been conducting an all-sky survey for stars with large proper motions using data from the Digitized Sky Surveys (DSS). The scanned images in the DSS cover the entire sky in multiple bands and at various epochs, and the temporal baseline between the earliest and latest epoch is between 15 and 45 years for most areas on the sky.

The large motion displayed by high proper motion stars between the two epochs is detected directly from the scans by means of an image subtraction algorithm, described in detail in Lépine et al.(2002). Two-epochs finder charts are generated, which can be blinked on the computer screen. All objects detected in the survey are thus verified by eye, and spurious detections are excluded.

At the bright end, stars tend to become saturated on the DSS images, and are no longer properly detected by the code. The TYCHO-2 catalog is used to complete the census at the bright end. Also, all stars detected by SUPERBLINK are searched for a counterpart in the TYCHO-2. The positional and proper motion information from the TYCHO-2 catalog is used for all matching counterparts. The two-epoch charts are however examined to verify consistency; in some cases, it is found that the proper motion from TYCHO-2 must be in error, and the SUPERBLINK proper motion is used instead.

Counterparts in the the 2MASS All-Sky Catalog of Point Sources (Cutri et al., 2003) are also identified for all SUPERBLINK detections. At the faint end, the positions are those extrapolated from the 2MASS catalog, and thus realized in the ICRS system and accurate to about 0.1". Counterpart are also identified in the USNO-B1 catalog (Monet et al., 2003), and together with 2MASS, provide optical and infrared magnitudes for almost all the stars.

The systematic comparison with the 2MASS catalog allows us to systematically identify all common proper motion doubles which are resolved in the 2MASS images. The CCD observations from 2MASS have significantly higher resolution than the 
photographic images from the DSS. Numerous common proper motion doubles and multiple systems have being identified and their components will be listed as separate entries in the LSPM catalog. Many faint companions of Hipparcos stars are also being identified this way (e.g. Lépine \& Bongiorno, 2007).
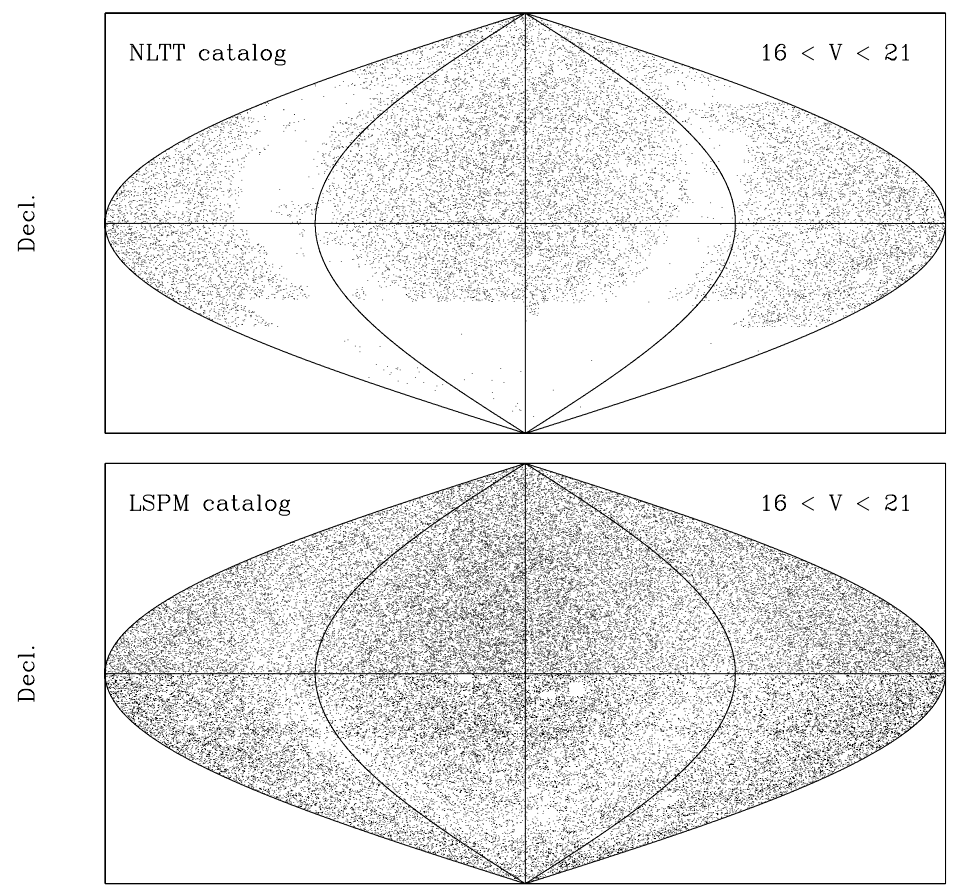

R.A.

Figure 1. Comparison between the distribution of faint $(V>16)$ stars from the NLTT catalog (top) and the new LSPM catalog (bottom). The new catalog fills in all the gaps of the NLTT, particularly in the low Galactic latitude fields, and provides the most complete all-sky census of high proper motion stars to date.

\section{Replacing the NLTT catalog}

The NLTT catalog (Luyten, 1979) was notoriously incomplete in two main regions: the sky south of Decl. $=-30^{\circ}$, and areas of high stellar density along the plane of the Milky Way. The incompleteness was most severe for stars fainter than magnitude $\mathrm{V}=16$ (see Fig.1).

The northern part of the SUPERBLINK survey was completed first, and the results have already been published in Lépine \& Shara(2005) as the LSPM-north catalog. The full LSPM catalog now fills in most of the remaining gaps in the south, and at last provides a true, all-sky census of faint stars with large proper motions (Fig.1). While there remains some level of incompleteness at low Galactic latitudes, especially toward the Galactic center, most of the variations in surface density observed in Fig.1 are due to selection effects from the high proper motion cutoff $\left(\mu>0.15^{\prime \prime} \mathrm{yr}^{-1}\right)$ of the survey. A combination of the Sun's motion through the local standard of rest and the asymmetric drift of the Galactic thick disk and halo stars results in more stars having large transverse motions at high Galactic latitudes, hence the larger density of stars detected there. 
Compared with the 58,845 stars with proper motions $\mu>0.18^{\prime \prime} \mathrm{yr}^{-1}$ listed in the NLTT catalog, the LSPM catalog will list over 122,000 stars with proper motions $\mu>0.18^{\prime \prime}$ $\mathrm{yr}^{-1}$. With the increased sky coverage and completeness, the LSPM catalog makes the Luyten catalog obsolete, and from now on should be used as a replacement to the NLTT. For convenience, all NLTT stars will be identified in the LSPM both by their LHS designation and NLTT catalog number.

\section{Stellar content and kinematics}

A reduced proper motion diagram shows the stars in the LSPM to be composed of three main classes. Low-mass $\mathrm{K}$ and $\mathrm{M}$ red dwarfs from the disk population dominate, but a significant fraction are low-mass subdwarfs from the halo (sdK, sdM), and the catalog also contains thousands of white dwarfs. While we currently lack parallax distances for most of the stars in the LSPM catalog, photometric distances can be calculated for specific classes of objects. The red dwarfs, in particular, have a reasonably well calibrated $\left[M_{v}, V-J\right]$ color-magnitude relationship (Lépine, 2005).

With photometric distances and proper motions, it is possible to investigate the local kinematics of the dwarfs in the vicinity of the Sun $(d<100 \mathrm{pc})$. By selecting stars in specific parts of the sky, one can obtain velocity-space projections in the UV, UW, and VW plane (Figure 2). Because of the high proper motion cutoff of the LSPM catalog $\left(\mu>0.15^{\prime \prime} \mathrm{yr}^{-1}\right)$, stars with low projected velocities are not represented in the census, which leaves a low-velocity "hole" in the maps of projected velocities. The hole increases for stars at larger distances. Despite this artifact, one can see that the velocity space projections of the nearby red dwarfs are not isotropic and show considerable structure. A comparison with the velocity space distribution of Hipparcos stars calculated by Nordström et al.(2004), shows very good agreement with our data. This shows how future astrometry, providing accurate parallaxes for all the LSPM stars, may have a major impact in uncovering fine structure in the kinematics of stars in the Solar vicinity.

\section{Conclusions}

The LSPM catalog now has all-sky coverage. The LSPM-south catalog will complement the already released LSPM-north, and yield a highly complete catalog of stars with proper motion $\mu>0.15^{\prime \prime} \mathrm{yr}^{-1}$. The catalog is estimated to be $>98 \%$ complete for all H-burning stars and white dwarfs with proper motions in the range above, covering virtually all objects down to visual magnitude 19. The catalog is realized at the bright end by the Tycho- 2 catalog, down to magnitude $\approx 10-12$. At the faint end, which encompass the vast majority of the stars, the proper motions are obtained from the SUPERBLINK software, while the positions are determined by the counterparts in the 2MASS catalog. Overall, the positional accuracy of the catalog is thus better than $0.12^{\prime \prime}$, while proper

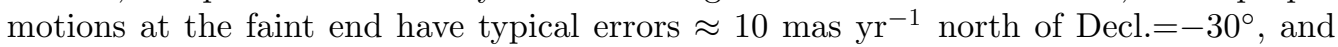
$\approx 20$ mas $_{\mathrm{yr}^{-1}}$ south of this. All double stars which are resolved in the 2MASS survey have been identified and are listed individually.

The SUPERBLINK survey is now being expanded to lower proper motion regimes,

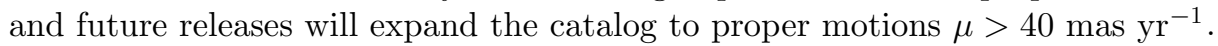



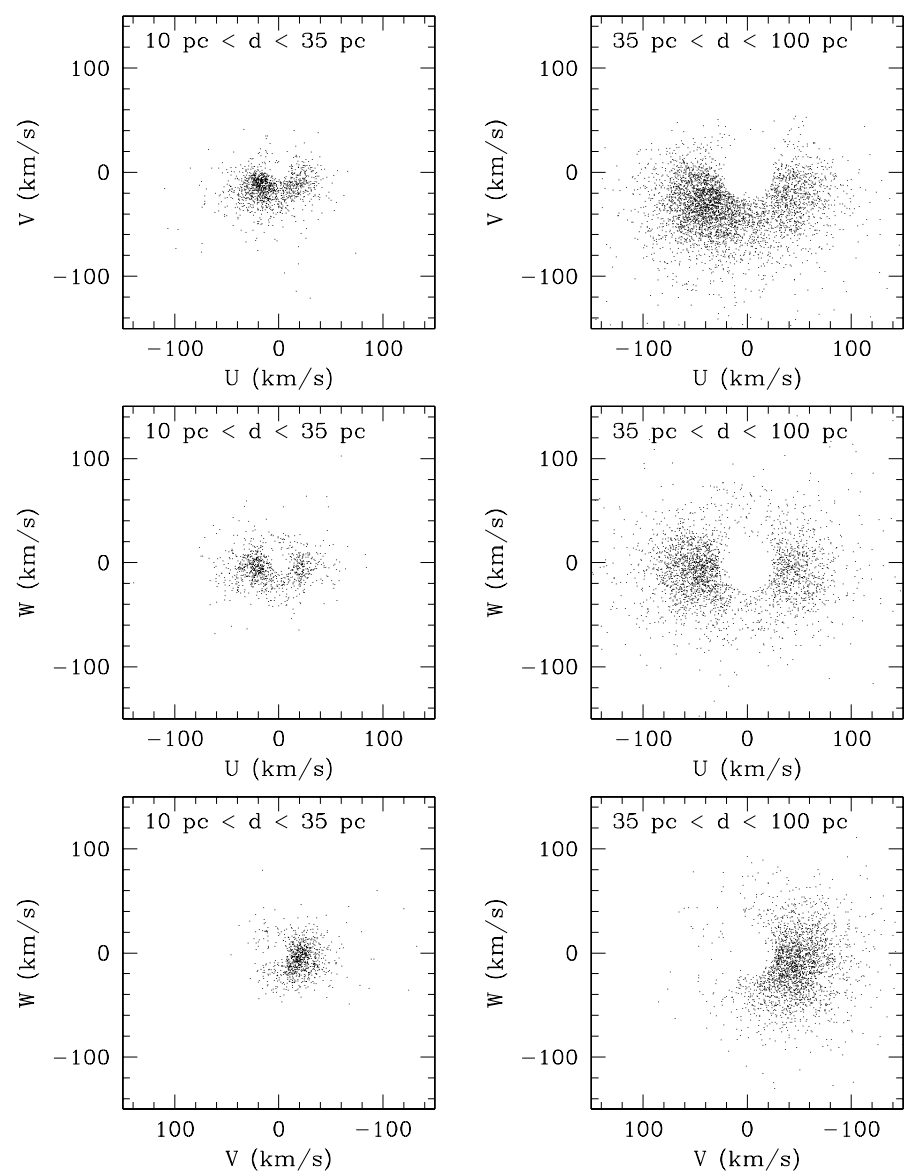

Figure 2. Projected motions in the UVW of red dwarf stars in the Solar neighborhood, based on LSPM catalog proper motions and photometric distances. The UW projection is from stars found at low Galactic latitude in the direction of the apex and antapex of the Sun's orbital motion around the Galaxy. The VW projection is obtained from stars in the direction of the Galactic center and anti-center.

\section{References}

Cutri, R. M., et al. 2003, The 2MASS All-Sky Catalog of Point Sources University of Massachusetts and Infrared Processing and Analysis Center (IPAC/California Institute of Technology.

Hog E., et al. 2000, A\&A A, 355, 27

Lépine, S., Shara, M. M., \& Rich R.M.R. 2003, $A J, 124,1190$

Lépine, S. \& Shara, M. M. 2005, AJ, 129, 1483

Lépine, S. 2005, AJ, 130, 1247

Lépine, S. \& Bongiorno, B. 2007, AJ, 133, 889

Luyten W. J. 1979, New Luyten Catalogue of stars with proper motions larger than two tenths of an arcsecond (NLTT), University of Minnesota, Minneapolis

Monet, D. G., et al. 2003, AJ, 125, 984

Nordström, B. et al. 2004, A\$A, 418, 989 